\title{
Regularity of attractor for 3D Ginzburg-Landau equation
}

\author{
Shujuan Lü, Qishao Lu, Qingguo Meng and Zhaosheng Feng
}

Communicated by Y. Charles Li, received April 1, 2009.

\begin{abstract}
In this paper, a three dimensional Ginzburg-Landau type equation with periodic initial value condition is considered. Firstly, the smoothing property of the solution is obtained by a uniform priori estimates; then, the existence of the global attractors, $\mathcal{A}_{i} \subset H_{p}^{i}(\Omega)(i=2,3, \cdots)$, of the semi-group $\left\{S^{(i)}(t)\right\}_{t \geq 0}$ of operators generated by the equation is presented by using the compactness principle; finally, the regularity of the global attractors is proved by the decomposition of semi-group.
\end{abstract}

\section{Contents}

1. Introduction 185

2. Existence of the Higher-order Attractors 188

3. Decomposition of Semi-group 192

4. Regularity of Attractor 199

$\begin{array}{ll}\text { References } & 200\end{array}$

\section{Introduction}

The Ginzburg-Landau-type equation, which arises in many mechanical, physical and chemical phenomena, is an important nonlinear evolution equation and has many concrete types. In particular, there is considerable attention on the Ginzburg-Landau equations (GLE) of type

$$
u_{t}-(1+\mathrm{i} \nu) \Delta u+(1+\mathrm{i} \mu)|u|^{2 \sigma} u-\gamma u=0,
$$

in one or two spatial dimension. For example, Ghidaglia and Héron [1], Doering et al. [2], Promislow [3], Bu [4] studied the finite-dimensional attractor and related

1991 Mathematics Subject Classification. 35B41, 35B45.

Key words and phrases. Ginzburg-Landau equation, Hölder's inequality, global attract, regularity of attractor, semi-group.

This work is supported by the National Natural Science Foundation of China (No.10872014) and UTPA Research Council Grant 119100. 
dynamical properties for 1D or 2D GLE (1.1) with nonlinearity where $\sigma=1$ or 2 . Lü [5] obtained the upper semi-continuity of approximation of attractors of (1.1) in $1 \mathrm{D}$ space with $\sigma=1$. For the dynamical behavior and asymptotical analysis of 2D GLE, some qualitative results have been established in [6-9]. Nevertheless, it seems that relevant results for the $3 \mathrm{D}$ case are very few. The main reason lies in the fact that some of the Sobolev interpolation inequalities used in $1 \mathrm{D}$ or $2 \mathrm{D}$ case become invalid in the $3 \mathrm{D}$ case. It is necessary to make more subtle estimates for nonlinear terms to overcome this difficulty. Doering at al. [10] considered the 3D case for equation (1.1) with periodic boundary conditions and proved the existence of global solutions under the following assumptions on $\sigma, \nu$ and $\mu$ :

$$
\left\{\begin{array}{cc}
|\nu|<\frac{2 \sqrt{3 \sigma-1}}{3 \sigma-2} \text { or } \frac{-\nu \mu}{|\nu-\mu|}<\frac{\sqrt{2 \sigma+1}}{\sigma}, & \text { if } \sigma<2 ; \\
|\nu|<\frac{2 \sqrt{3 \sigma-1}}{3 \sigma-2}, & \text { if } \sigma \geq 2 .
\end{array}\right.
$$

Okazawa and Yokota [11] proved the global existence of unique solutions for equation (1.1) with initial boundary value conditions under the assumption:

$$
|\nu| \leq \frac{\sqrt{2 \sigma+1}}{\sigma}
$$

In $[12,13]$, Lü and $\mathrm{Lu}$ discussed the initial-value problem of the following Ginzburg-Landau type equation with the spatial periodicity condition in the threedimensional space:

$$
\begin{gathered}
u_{t}-(1+\mathrm{i} \nu) \Delta u+(1+\mathrm{i} \mu)|u|^{2 \sigma} u-\gamma u=0, x \in \mathbf{R}^{3}, \quad t \in \mathbf{R}^{+}, \\
u(x, 0)=u_{0}(x), \quad x \in \mathbf{R}^{\mathbf{3}}, \\
u\left(x+\mathrm{e}_{j}, t\right)=u(x, t), \quad j=1,2,3, \quad t \in \mathbf{R}^{+},
\end{gathered}
$$

where $\nu, \mu, \gamma$ are real constants and $\gamma>0$, and they satisfy

$$
\left\{\begin{array}{lll}
\frac{-1-\nu \mu}{|\nu-\mu|}<\frac{\sqrt{2 \sigma+1}}{\sigma} & \text { or } \quad|\nu|<\frac{2 \sqrt{3 \sigma-1}}{3 \sigma-2}, & \text { if } \sigma \leq 2 ; \\
|\mu|<\frac{\sqrt{2 \sigma+1}}{\sigma} \quad \text { or } & |\nu|<\frac{2 \sqrt{3 \sigma-1}}{3 \sigma-2}, & \text { if } \sigma>2 .
\end{array}\right.
$$

In [12], the existences of the unique solution, finite-dimensional global attractor and exponential attractor was obtained, and the upper semi-continuity of global attractor was established in [13]. A global compact attractor and asymptotic behaviors for high-dimensional defocusing nonlinear systems were presented in $[14$, $15]$.

It is notable that condition (A3) improves that of (A1) and (A2). This can be clearly seen from the curves illustrated in Figure 1.

In this paper, we wish to further consider the regularity of global attractors for problem (1.2)-(1.4). The regularity is to be understood here in the sense of partial differential equations, i.e., if the date is sufficiently regular, then the global attractor lies in a Sobolev space $H_{p}^{m}(\Omega)$ for an appropriate number $m$.

The rest of this paper is organized as follows. In Section 2, the smoothing property of solutions is obtained by a priori estimate (see Lemma 2.2), and then the existence of the global attractors $\mathcal{A}_{i} \subset H_{p}^{i}(\Omega)(i=3,4, \cdots m)$ for the semi-group 


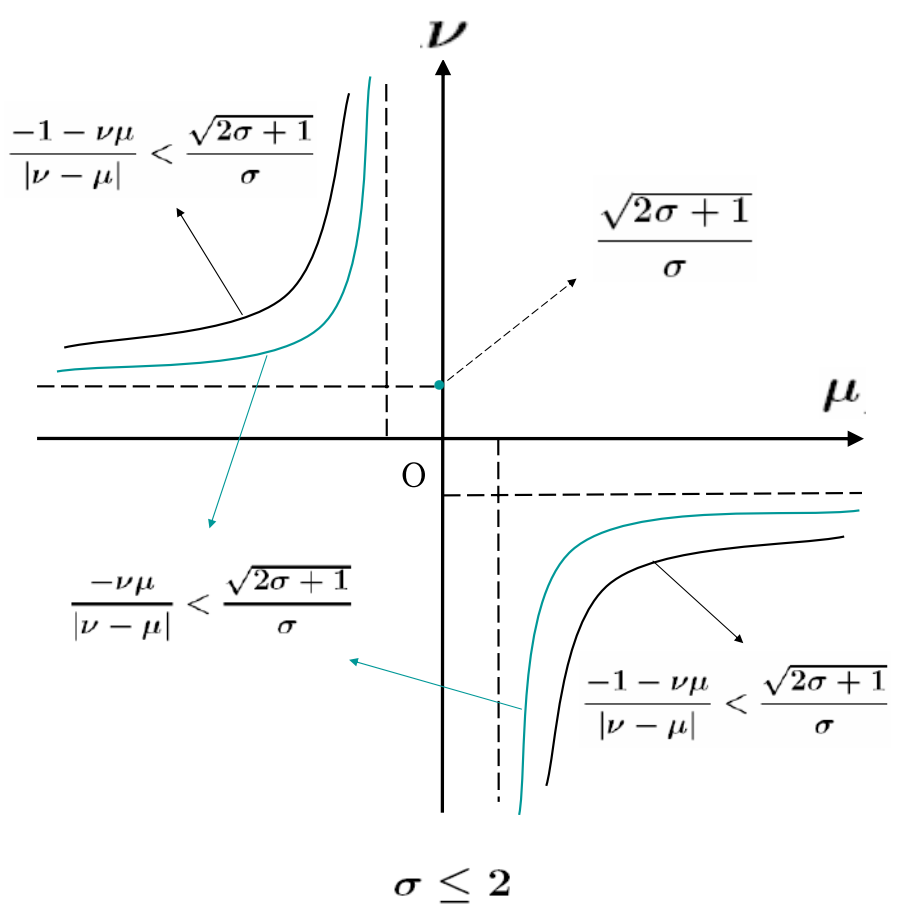

Figure 1. Two curves in (A1) and (A3).

of operators, $\left\{S^{(i)}(t)\right\}_{t>0}$, generated by problem (1.2)-(1.4) is proved (see Theorem 2.4). In Section 3, the solution operator $S^{(2)}(t)$ is decomposed as $S_{1}^{(2)}(t)+S_{2}^{(2)}(t)$, where $S_{1}^{(2)}(t) u_{0}$ is more regular than $S^{(2)}(t) u_{0}$, and $\left\|S_{2}^{(2)} u_{0}\right\|_{2}$ approaches zero as $t$ tends to infinity, uniformly for $u_{0}$ bounded in $H_{p}^{2}(\Omega)$ (see Theorem 3.2). In Section 4 we present the result on regularity of global attractors, namely, $\mathcal{A}_{2}=\mathcal{A}_{3}=\cdots=$ $\mathcal{A}_{m}$ (see Theorem 4.1).

Throughout this paper we will use the following notations: $\Omega=[0,1] \times[0,1] \times$ $[0,1],(\cdot, \cdot)$ denotes the usual inner product of $L^{2}(\Omega),\|\cdot\|_{m}$ denotes the norm of Sobolev spaces $H^{m}(\Omega),\|\cdot\|=\|\cdot\|_{0}$ and $\|\cdot\|_{\infty}=\|\cdot\|_{L^{\infty}(\Omega)}$. Let $L_{p}^{2}(\Omega)=\{\phi \in$ $\left.L^{2}(\Omega) \mid \phi\left(x+\mathbf{e}_{j}\right)=\phi(x), j=1,2,3\right\}$ with the norm defined just as that of $L^{2}(\Omega)$. Let $H_{p}^{m}(\Omega)=\left\{\phi \in H_{p}^{2}(\Omega) \mid \phi\left(x+\mathbf{e}_{j}\right)=\phi(x), j=1,2,3\right\}$ with the norm defined just as that of $H^{2}(\Omega)$.

In order to present our results in a straightforward manner, we need the following three technical lemmas:

LEMMA 1.1. [16] (Sobolev interpolation inequality) Suppose that $u \in L^{q}(\Omega)$, $D^{m} u \in L^{r}(\Omega), \Omega \subset R^{n}, 1 \leq r \leq \infty, 0 \leq j \leq m$. Then there exists a constant $c=c(j, m, \Omega, p, q, r)$ independent of $u$ such that

$$
\left\|D^{j} u\right\|_{L^{p}} \leq c\|u\|_{W^{m, r}(\Omega)}^{a}\|u\|_{L^{q}}^{1-a}
$$


where

$$
\frac{1}{p}=\frac{j}{n}+a\left(\frac{1}{r}-\frac{m}{n}\right)+(1-a) \frac{1}{q}, \quad \frac{j}{m}<a<1 .
$$

LEMMA 1.2. [17] (uniform Gronwall's inequality) Let $y(t), g(t)$ and $h(t)$ be three non-negative functions satisfying

$$
y^{\prime}(t) \leq g(t) y(t)+h(t), \quad \forall t \geq t_{0} \geq 0,
$$

and

$$
\int_{t}^{t+r} g(s) \mathrm{d} s \leq \alpha_{1}, \quad \int_{t}^{t+r} h(s) \mathrm{d} s \leq \alpha_{2}, \quad \int_{t}^{t+r} y(s) \mathrm{d} s \leq \alpha_{3}, \quad \forall t \geq t_{0} .
$$

Then we have

$$
y(t+r) \leq\left(\frac{\alpha_{3}}{r}+\alpha_{2}\right) \mathrm{e}^{\alpha_{1}}, \quad \forall t \geq t_{0} .
$$

LEMMA 1.3. [18] Let $\mathcal{E}$ be a Banach space, and suppose that $\{S(t)\}_{t \geq 0}$ is a semi-group of continuous operators, i.e., $S(t): \mathcal{E} \rightarrow \mathcal{E}$, with

$$
S(t) \cdot S(\tau)=S(t+\tau), \quad S(0)=I,
$$

where $I$ is the identify operator. We also suppose that the operator $S(t)$ satisfies that

(i) operator $S(t)$ is bounded, i.e., for any given $R>0$, if $\|u\|_{\mathcal{E}} \leq R$, then there exists a constant $C(R)$ such that

$$
\|S(t) u\|_{\mathcal{E}} \leq C(R), \quad \text { for } t \in[0,+\infty)
$$

(ii) there is a bounded absorbing set $\mathcal{B}_{0} \subset \mathcal{E}$, i.e., for any given bounded set $\mathcal{B} \subset \mathcal{E}$, there exists a constant $T=T(\mathcal{B})$ such that

$$
S(T) \mathcal{B} \subset \mathcal{B}_{0}, \quad \text { for } t \geq T
$$

(iii) $S(t)$ is a completely continuous operator for $t>0$ sufficiently large.

Then, the semi-group $\{S(t)\}_{t \geq 0}$ of operators has a compact global attractor $\mathcal{A} \subset \mathcal{E}$.

This lemma will help us better understand the proof of Theorem 4.1.

\section{Existence of the Higher-order Attractors}

In this section, we prove the existence of the global attractor $\mathcal{A}_{m} \subset H_{p}^{m}(\Omega)$ $(m=2,3, \cdots)$. Hence, the high-order smoothness of the solution is a prerequisite. To this end, we need the following lemma:

LEMma 2.1. [12] Suppose that condition (A3) holds and $u_{0}(x) \in H_{p}^{2}(\Omega)$. Then problem (1.2)-(1.4) possesses a unique global solution $u(x, t) \in L^{\infty}\left(R^{+} ; H_{p}^{2}(\Omega)\right) \cap$ $L^{2}\left([0, T] ; H_{p}^{3}(\Omega)\right)$, and for any $R>0$ given, there exists a value $t_{2}=t_{2}(R)$ such that

$\|u\|_{2} \leq E_{2}, \quad \forall t \geq 0$ and $u_{0}$ satisfying $\left\|u_{0}\right\|_{2} \leq R$,

$\|u\|_{2} \leq M_{2}, \quad \forall t \geq t_{2}$ and $u_{0}$ satisfying $\left\|u_{0}\right\|_{2} \leq R$,

where the constant $E_{2}$ depends on the parameters $\sigma, \nu, \mu, \gamma$ and $R$, and $M_{2}$ only depends on the parameters $\sigma, \nu, \mu$ and $\gamma$.

Furthermore, when $\sigma \geq \frac{1}{2}$, then the semi-group $\{S(t)\}_{t \geq 0}$ of operators generated by problem (1.2)-(1.4) has a compact global attractor $\mathcal{A}_{2} \triangleq \mathcal{A} \subset H_{p}^{2}(\Omega)$, i.e., 
there exists a set $\mathcal{A} \subset H_{p}^{2}(\Omega)$ such that

(a) $S(t) \mathcal{A}=\mathcal{A}$ for all $t \geq 0$;

(b) $\operatorname{dist}(S(t) \mathcal{B}, \mathcal{A}) \rightarrow 0$ for any bounded set $\mathcal{B} \subset H_{p}^{2}(\Omega)$, where

$$
\operatorname{disp}(X, Y)=\sup _{x \in X} \inf _{y \in Y}\|x-y\|_{2} .
$$

Using this lemma, we can now show a property of the high-order smoothness of the global solution for problem (1.2)-(1.4).

LEMMA 2.2. Under the conditions of Lemma 2.1, suppose that $m \geq 2$ is a given positive integer, and $\sigma \geq \frac{1}{2}\left(\left[\frac{m}{2}\right]-1\right)$ or $\sigma$ is a positive integer. Then there exists $t_{m}=t_{m}(R)$ such that

$$
\|u\|_{m} \leq M_{m} \text { for all } t \geq t_{m} \text { if }\left\|u_{0}\right\|_{2} \leq R,
$$

and

$$
\|u\|_{m} \leq E_{m} \text { for all } t \geq 0 \text { if }\left\|u_{0}\right\|_{m} \leq R,
$$

where the constant $E_{m}$ depends on the parameters $\sigma, \nu, \mu, \gamma, m$ and $R$, and $M_{m}$ only depends on the parameters $\sigma, \nu, \mu, \gamma$ and $m$.

Thus, problem (1.2)-(1.4) possesses the global smooth solution $u \in C\left(R^{+} ; H_{p}^{m}(\Omega)\right) \cap$ $C^{1}\left(R^{+} ; H_{p}^{m-2}(\Omega)\right)$, and the closed ball

$$
B_{m}=\left\{\varphi \in H_{p}^{m}(\Omega) \mid\|\varphi\|_{m} \leq M_{m}\right\},
$$

is a bounded absorbing set of the semi-group of operators $\left\{S^{(m)}(t)\right\}_{t \geq 0}$.

Proof. We prove (2.1) and (2.2) by using mathematical induction. Apparently, for $m=2,(2.1)$ and $(2,2)$ can be deduced by Lemma 2.1 immediately.

We suppose that (2.1) and (2.2) hold for $m=2,3, \cdots, k-1 \geq 3$, where $k$ is a positive integer, i.e.

$$
\|u\|_{m} \leq M_{m} \text { for all } t \geq t_{m} \text { if }\left\|u_{0}\right\|_{2} \leq R, \quad m=2,3, k-1,
$$

$$
\|u\|_{m} \leq E_{m} \text { for all } t \geq 0 \text { if }\left\|u_{0}\right\|_{m} \leq R, \quad m=2,3, k-1 .
$$

Then by using the Sobolev interpolation inequality (see Lemma 1.1), there exist constants $E_{m}^{\prime}=E_{m}^{\prime}(R)$ and $M_{m}^{\prime}$ such that

$$
\|u\|_{W^{m-2, \infty}} \leq M_{m}^{\prime}, \quad \forall t \geq t_{m}, \quad 3 \leq m \leq k-1,
$$

and

$$
\|u\|_{W^{m-2, \infty}} \leq E_{m}^{\prime}, \quad \forall t \geq 0, \quad 3 \leq m \leq k-1 .
$$

We shall prove that both (2.1) and (2.2) hold for $m=k$.

Taking $l=\left[\frac{k-1}{2}\right]$ and differentiating (1.2) for $l$ times with respect to $t$, we have

$$
u_{t^{l+1}}-(1+\mathrm{i} \nu) \triangle u_{t^{l}}+(1+\mathrm{i} \mu)\left(|u|^{2 \sigma} u\right)_{t^{l}}-\gamma u_{t^{l}}=0 .
$$

If $k=2 l+1$ is an odd number, then the real part of the $L^{2}$-inner product of $(2.7)$ with $-\triangle u_{t^{l}}$ is

$$
\frac{1}{2} \frac{\mathrm{d}}{\mathrm{d} t}\left\|\nabla u_{t^{l}}\right\|^{2}+\left\|\triangle u_{t^{l}}\right\|^{2}=\gamma\left\|\nabla u_{t^{l}}\right\|^{2}+\operatorname{Re}\left((1+\mathrm{i} \mu) \int_{\Omega}\left(|u|^{2 \sigma} u\right)_{t^{l}} \triangle \bar{u}_{t^{l}} \mathrm{~d} x\right) .
$$


For $t \geq t_{k-1}=t_{2 l}, \sigma \geq \frac{1}{2}(l-1)=\frac{1}{2}\left(\left[\frac{k}{2}\right]-1\right)$ or $\sigma$ is a positive integer, in view of Hölder's inequality, the Sobolev interpolation inequality and Young's inequality together with $(2.3)-(2.6)$, for the last two terms in (2.8) we have

$$
\begin{gathered}
\left|(1+\mathrm{i} \mu) \int_{\Omega}\left(|u|^{2 \sigma} u\right)_{t^{l}} \triangle \bar{u}_{t^{l}} \mathrm{~d} x\right| \leq \frac{1}{4}\left\|\Delta u_{t^{l}}\right\|^{2}+c\left(M_{k-1}, M_{k-1}^{\prime}\right), \\
\gamma\left\|\nabla u_{t^{l}}\right\|^{2} \leq \gamma\left\|\triangle u_{t^{l}}\right\|\left\|u_{t^{l}}\right\| \leq \frac{1}{4}\left\|\left.\triangle u_{t^{l}}\right|^{2}+\gamma^{2}\right\| u_{t^{l}} \|^{2} .
\end{gathered}
$$

Thus, equation (2.8) can be rewritten as

$$
\frac{\mathrm{d}}{\mathrm{d} t}\left\|\nabla u_{t^{l}}\right\|^{2}+\left\|\triangle u_{t^{l}}\right\|^{2} \leq C_{1}\left(M_{k-1}^{\prime}, M_{k-1}\right), \quad \forall t \geq t_{k-1}
$$

Taking the $L^{2}$-inner product of $(2.7)$ with $u_{t^{l}}$ and using a similar procedure to the deduction of (2.9), we obtain

$$
\frac{\mathrm{d}}{\mathrm{d} t}\left\|u_{t^{l}}\right\|^{2}+\left\|\nabla u_{t^{l}}\right\|^{2} \leq C_{2}\left(M_{k-1}, M_{k-1}^{\prime}\right), \quad \forall t \geq t_{k-1} .
$$

Thanks to $(2.10)$, it is found that

$$
\begin{aligned}
\int_{t}^{t+1}\left\|\nabla u_{t^{l}}(s)\right\|^{2} \mathrm{~d} s & \leq\left\|u_{t^{l}}(t)\right\|^{2}+C_{2}\left(M_{k-1}^{\prime}, M_{k-1}\right), \\
& \leq C\left(M_{k-1}, M_{k-1}^{\prime}\right)+C_{2}\left(M_{k-1}^{\prime}, M_{k-1}\right) \triangleq \alpha_{3}, \quad \forall t \geq t_{k-1} .
\end{aligned}
$$

In addition, we set

$$
\alpha_{2}=C_{1}\left(M_{k-1}^{\prime}, M_{k-1}\right) .
$$

Applying the uniform Gronwall's inequality (see Lemma 1.2) to (2.9), we have

$$
\left\|\nabla u_{t^{l}}(t+1)\right\|^{2} \leq \alpha_{3}+\alpha_{2}, \quad \forall t \geq t_{k-1}
$$

which leads to

$$
\left\|\nabla^{k} u\right\|^{2}=\left\|\nabla^{2 l+1} u\right\|^{2} \leq C\left(\alpha_{2}, \alpha_{3}\right), \quad \forall t \geq t_{k-1}+1 .
$$

Let $M_{k}=\sqrt{C\left(\alpha_{2}, \alpha_{3}\right)+M_{k-1}^{2}}$ and $t_{k}=t_{k-1}+1$, then (2.1) holds for $m=k$.

If $u_{0} \in H_{p}^{k}(\Omega)$, using a similar procedure to the deduction of (2.9), then equation $(2.8)$ can be rewritten as

$$
\frac{\mathrm{d}}{\mathrm{d} t}\left\|\nabla u_{t^{l}}\right\|^{2}+\left\|\nabla u_{t^{l}}\right\|^{2}+\left\|\triangle u_{t^{l}}\right\|^{2} \leq C_{3}\left(E_{k-1}^{\prime}, E_{k-1}\right), \quad \forall t \geq 0 .
$$

Multiplying the above expression by $\mathrm{e}^{t}$ and integrating it with respect to $t$, we deduce

$$
\left\|\nabla u_{t^{l}}\right\|^{2} \leq \mathrm{e}^{-t}\left\|\nabla u_{t^{l}}(0)\right\|^{2}+C_{3} \leq C(R)+C_{3}\left(E_{k-1}^{\prime}, E_{k-1}\right), \quad \forall t \geq 0,
$$

which leads to

$$
\left\|\nabla^{k} u\right\|^{2}=\left\|\nabla^{2 l+1} u\right\|^{2} \leq C\left(C_{3}, C\right) \triangleq E_{k}^{\prime \prime} .
$$

Let $E_{k}=\sqrt{E_{k}^{\prime \prime}+E_{k-1}^{2}}$, then (2.2) holds for $m=k$.

If $k=2 l+2$ is an even number, then taking the $L^{2}$-inner products of (2.7) with $\triangle^{2} u_{t^{l}}$ and $-\triangle u_{t^{l}}$, respectively, and similar to the deduction of (2.9) and (2.10), we can obtain

$$
\frac{\mathrm{d}}{\mathrm{d} t}\left\|\triangle u_{t^{l}}\right\|^{2}+\left\|\nabla \triangle u_{t^{l}}\right\|^{2} \leq C_{1}^{\prime}\left(M_{k-1}, M_{k-1}^{\prime}\right), \quad \forall t \geq t_{k-1},
$$


and

$$
\frac{\mathrm{d}}{\mathrm{d} t}\left\|\nabla u_{t^{l}}\right\|^{2}+\left\|\triangle u_{t^{l}}\right\|^{2} \leq C_{2}^{\prime}\left(M_{k-1}, M_{k-1}^{\prime}\right), \quad \forall t \geq t_{k-1},
$$

respectively. Using the same arguments as that of (2.11), we have

$$
\left\|\triangle u_{t^{l}}(t+1)\right\|^{2} \leq \alpha_{3}^{\prime}+\alpha_{2}^{\prime}, \quad \forall t \geq t_{k-1},
$$

which leads to

$$
\left\|\nabla^{k} u\right\|^{2}=\left\|\nabla^{2 l+2} u\right\|^{2} \leq C\left(\alpha_{2}^{\prime}, \alpha_{3}^{\prime}\right), \quad \forall t \geq t_{k-1}+1 .
$$

Let $M_{k}=\sqrt{C\left(\alpha_{2}^{\prime}, \alpha_{3}^{\prime}\right)+M_{k-1}^{2}}$ and $t_{k}=t_{k-1}+1$, then $(2.1)$ holds for $m=k$.

If $u_{0} \in H_{p}^{k}(\Omega)$, then similar to the deduction of (2.12) and (2.13), it is derived that

$$
\frac{\mathrm{d}}{\mathrm{d} t}\left\|\triangle u_{t^{l}}\right\|^{2}+\left\|\triangle u_{t^{l}}\right\|^{2}+\left\|\nabla \triangle u_{t^{l}}\right\|^{2} \leq C_{3}^{\prime}\left(E_{k-1}, E_{k-1}^{\prime}\right), \quad \forall t \geq 0 .
$$

Multiplying the above expression by $\mathrm{e}^{t}$ and integrating it with respect to $t$, we have

$$
\left\|\triangle u_{t^{l}}\right\|^{2} \leq \mathrm{e}^{-t}\left\|\triangle u_{t^{l}}(0)\right\|^{2}+C_{3}^{\prime}\left(E_{k-1}, E_{k-1}^{\prime}\right) \leq C\left(R, E_{k-1}, E_{k-1}^{\prime}\right), \quad \forall t \geq 0,
$$

which leads to

$$
\left\|\nabla^{k} u\right\|^{2}=\left\|\nabla^{2 l+2} u\right\|^{2} \leq E_{k}^{\prime \prime}\left(R, E_{k-1, E_{k-1^{\prime}}}\right) \triangleq E_{k}^{\prime \prime}, \quad \forall t \geq 0 .
$$

Let $E_{k}=\sqrt{E_{k-1}^{2}+E_{k}^{\prime \prime}}$, then (2.2) holds for $m=k$.

By means of mathematical induction, both (2.1) and (2.2) hold for any positive integer $m \geq 2$. Consequently, the proof of Lemma 2.2 is completed.

In order to obtain the existence of the high-order global attractor $\mathcal{A}_{m}$, we introduce the following technical result:

LEMMA 2.3. Suppose that the conditions of Lemma 2.2 hold with $\sigma \geq \frac{1}{2}\left(\left[\frac{m+1}{2}\right]-\right.$ $1)$ or a positive integer. Then the semi-group of operations $S^{m}(t)(t \geq 0)$ : $H_{p}^{m}(\Omega) \rightarrow H_{p}^{m}(\Omega)$ is uniformly compact for sufficiently large $t>0$.

PROOF. If $m=2 l$ is an even number, then taking the real parts of the inner products of $(2.7)$ with $-\triangle u_{t^{l}}$ and $u_{t^{l}}$, respectively, and applying Lemma 2.2 , we find that there exist constants $C_{1}=C_{1}\left(M_{m}, M_{m}^{\prime}\right)$ and $C_{2}=C_{2}\left(M_{m}, M_{m}^{\prime}\right)$ such that

$$
\frac{\mathrm{d}}{\mathrm{d} t}\left\|\nabla u_{t^{l}}\right\|^{2}+\left\|\triangle u_{t^{l}}\right\|^{2} \leq C_{1}\left(M_{m}^{\prime}, M_{m}\right), \quad \forall t \geq t_{m}
$$

and

$$
\frac{\mathrm{d}}{\mathrm{d} t}\left\|u_{t^{l}}\right\|^{2}+\left\|\nabla u_{t^{l}}\right\|^{2} \leq C_{2}\left(M_{m}, M_{m}^{\prime}\right), \quad \forall t \geq t_{m}
$$

Applying the uniform Gronwall's inequality to (2.14), using (2.15) and Lemma 2.2, we obtain

which leads to

$$
\left\|\nabla u_{t^{l}}\right\|^{2} \leq C_{3}\left(M_{m}, M_{m}^{\prime}\right), \quad \forall t \geq t_{m+1}=t_{m}+1
$$

$\left\|\nabla^{m+1} u\right\|^{2}=\left\|\nabla^{2 l+1} u\right\|^{2} \leq C\left(M_{m}, M_{m}^{\prime}\right)\left\|\nabla u_{t^{l}}\right\|^{2} \leq M_{m+1}, \quad \forall t \geq t_{m+1}=t_{m}+1$.

If $m=2 l$ is an odd number, then using the similar arguments to that of (2.11) and (2.12), we obtain

$$
\frac{\mathrm{d}}{\mathrm{d} t}\left\|u_{t^{l}}\right\|^{2}+\left\|\nabla u_{t^{l}}\right\|^{2} \leq C_{1}^{\prime}\left(M_{m}, M_{m}^{\prime}\right)\left(\left\|u_{t^{l}}\right\|^{2}+1\right), \quad \forall t \geq t_{m}
$$


and

$$
\frac{\mathrm{d}}{\mathrm{d} t}\left\|\nabla u_{t^{l-1}}\right\|^{2}+\left\|\triangle u_{t^{l-1}}\right\|^{2} \leq C_{2}^{\prime}\left(M_{m}, M_{m}^{\prime}\right), \quad \forall t \geq t_{m}
$$

Again, applying the uniform Gronwall's inequality to (2.16) as well as (2.17), we find

$$
\left\|\nabla^{m+1} u\right\|^{2}=\left\|\nabla^{2 l} u\right\|^{2} \leq C\left(M_{m}, M_{m}^{\prime}\right)\left\|u_{t^{l}}\right\|^{2} \leq M_{m+1}, \quad \forall t \geq t_{m+1}=t_{m}+1 .
$$

By using the Sobolev compact imbedding theorem, we know that the semigroup of operators $S^{(m)}(t)$ is uniformly compact for $t \geq t_{m+1}$. So the proof of Lemma 2.3 is completed.

On the other hand, if $\sigma \geq \frac{1}{2}\left(\left[\frac{m}{2}\right]\right)$ or $\sigma>0$ is an integer, then similar to Lemmas 2.2 and 2.3 , it can be proved that $S^{(m)}(t)$ is strongly continuous. Therefore, by virtue of Lemmas 2.2 and 2.3 , we obtain the main result immediately in this section as follows:

ThEOREM 2.4. Suppose that all conditions of Lemma 2.3 hold. Then there exists a global attractor $\mathcal{A}_{m} \subset H_{p}^{m}(\Omega)$ of the semi-group $\left\{S^{(m)}(t)\right\}_{t \geq 0}$ of the operators generated by problem (1.2)-(1.4).

\section{Decomposition of Semi-group}

In order to prove the regularity of global attractor, it is necessary to decompose $S^{(2)}(t)$ appropriately. In this section, we decompose $S^{(2)}(t)$ as $S_{1}^{(2)}(t)+S_{2}^{(2)}(t)$, where $S_{1}^{(2)}(t) u_{0}$ is more regular than $S^{(2)}(t) u_{0}$, and $\left\|S_{2}^{(2)}(t) u_{0}\right\|_{2}$ approaches zero as $t$ goes to infinity, uniformly for $u_{0}$ bounded in $H_{p}^{2}(\Omega)$.

For any given positive integer $N$, let $S_{N}=\operatorname{Span}\left\{\mathrm{e}^{2 \pi \mathrm{i} k \cdot x}:|k| \leq N\right\}$ and denote the orthogonal projection operators by $P_{N}: L_{p}^{2}(\Omega) \rightarrow S_{N}$ and $Q_{N}=I-P_{N}$ (see [19]). Then we have

LEMMA 3.1. [19] If $v \in H_{p}^{m}(\Omega)$, then there exists a constant $c$ independent of $v$ and $N$ such that

$$
\begin{gathered}
\left\|P_{N} v\right\|_{m} \leq c N^{m-j}\left\|P_{N} v\right\|_{j}, \quad \forall 0<j \leq m, \\
\left\|Q_{N} v\right\|_{j} \leq c N^{j-m}\left\|Q_{N} v\right\|_{m}, \quad \forall j=0,1, \cdots, m,
\end{gathered}
$$

and

$$
\left\|\nabla^{j} Q_{N} v\right\| \leq c N^{j-m}\left\|\nabla^{m} Q_{N} v\right\|, \quad \forall j=0,1, \cdots, m .
$$

We now decompose the solution $u(x, t)$. Suppose that $u_{0} \in H_{p}^{2}(\Omega)$ and $u=$ $S(t) u_{0}$ is the solution of problem (1.2)-(1.4), then we have

$$
u=P_{N} u+Q_{N} u=p(t)+q(t),
$$

where $p(t)=P_{N} u$ is the low-frequency part of $u$ and $q(t)=Q_{N} u$ is the highfrequency part of $u$.

We split the high-frequency part $q(t)$ as

$$
q(t)=y+z,
$$

where $y, z \in Q_{N} L_{p}^{2}(\Omega)$ are the solutions of the following equations for $t \geq t_{2}$ :

$$
\begin{gathered}
y_{t}-\gamma y-(1+\mathrm{i} \nu) \Delta y+(1+\mathrm{i} \mu) Q_{N}\left(|u|^{2 \sigma}(p+y)\right)=0, \\
y(x, t)=y\left(x+\mathrm{e}_{j}, t\right), \quad j=1,2,3 ; \quad y\left(x, t_{2}\right)=0,
\end{gathered}
$$


and

$$
\begin{gathered}
z_{t}=\gamma z+(1+\mathrm{i} \nu) \Delta z-(1+\mathrm{i} \mu) Q_{N}\left(|u|^{2 \sigma} z\right), \\
z(x, t)=z\left(x+\mathrm{e}_{j}, t\right), \quad j=1,2,3 ; \quad z\left(x, t_{2}\right)=Q_{N} u\left(t_{2}\right),
\end{gathered}
$$

respectively. For $t \leq t_{2}$, we have that $y(t)=0$ and $z(t)=Q_{N} u(t)$, where $t_{2}$ is given as that in Lemma 2.2.

Next, we prove that $y$ is smooth for $t \geq t_{m}$ and $z$ converges toward 0 in $H_{p}^{2}(\Omega)$ when $t$ goes to infinity.

THEOREM 3.2. Under condition (A3), if $u_{0} \in H_{p}^{2}(\Omega)$ satisfies $\left\|u_{0}\right\|_{2} \leq R$, then there exist a unique solution $y$ of (3.1) and (3.2), and a unique solution $z$ of (3.3) and (3.4), which satisfy

$$
y, z \in C^{1}\left([0, \infty) ; L_{p}^{2}(\Omega)\right) \cap C\left([0, \infty) ; H_{p}^{2}(\Omega)\right),
$$

respectively. Moreover, there exist a number $N_{3}$ large enough, constants $K_{m}=$ $K_{m}(N)$ and $\lambda=\lambda(N)>0$ such that for any given $N \geq N_{3}$, the following estimates hold:

$$
\begin{gathered}
\|y(t)\|_{m} \leq K_{m}, \quad \forall t \geq t_{m}, \quad m=2,3, \cdots, \\
\|z(t)\|_{2} \leq C\left(M_{2}\right) \mathrm{e}^{-\lambda\left(t-t_{2}\right)}, \quad \forall t \geq t_{2},
\end{gathered}
$$

where $M_{2}, t_{m}$ and $R$ are given as that in Lemmas 2.1-2.2.

Proof. The existence and uniqueness can be proved by using the usual Galerkin methods $[12,13]$, so we omit it. In what follows, we separate our proof of estimates (3.5) and (3.6) into three steps.

Step 1: estimate for $y$ in $H_{p}^{2}(\Omega)$.

Since the real part of the inner product of (3.1) with $y$ is

$$
\frac{1}{2} \frac{\mathrm{d}}{\mathrm{d} t}\|y\|^{2}+\|\nabla y\|^{2}-\gamma\|y\|^{2}+\operatorname{Re}\left((1+\mathrm{i} \mu)\left(Q_{N}\left(|u|^{2 \sigma}(p+y)\right), y\right)\right)=0,
$$

for the last term in the left-hand side of the above equation, from the definition of $Q_{N}$ we have

$$
\operatorname{Re}(1+\mathrm{i} \mu)\left(Q_{N}\left(|u|^{2 \sigma}(p+y), y\right)=\int_{\Omega}|u|^{2 \sigma}|y|^{2} \mathrm{~d} x+\operatorname{Re}\left((1+\mathrm{i} \mu) \int_{\Omega}|u|^{2 \sigma} p \bar{y} \mathrm{~d} x\right) .\right.
$$

By applying Hölder's inequality, Young's inequality, the Sobolev interpolation inequality and Lemma 2.2, we can obtain

$$
\begin{aligned}
\operatorname{Re}\left((1+\mathrm{i} \mu) \int_{\Omega}|u|^{2 \sigma} p \bar{y} \mathrm{~d} x\right) & \leq|1+\mathrm{i} \mu|\left(\int_{\Omega}|u|^{2 \sigma}|y|^{2} \mathrm{~d} x\right)^{\frac{1}{2}}\left(\int_{\Omega}|u|^{2 \sigma}|p|^{2} \mathrm{~d} x\right)^{\frac{1}{2}}, \\
& \leq \frac{1}{2} \int_{\Omega}|u|^{2 \sigma}|y|^{2} \mathrm{~d} x+\frac{|1+\mathrm{i} \mu|^{2}}{2} \int_{\Omega}|u|^{2 \sigma}|p|^{2} \mathrm{~d} x \\
& \leq \frac{1}{2} \int_{\Omega}|u|^{2 \sigma}|y|^{2} \mathrm{~d} x+C\left(M_{2}\right) .
\end{aligned}
$$

Substituting this inequality into equation (3.7) yields

$$
\frac{\mathrm{d}}{\mathrm{d} t}\|y\|^{2}+2\|\nabla y\|^{2}-2 \gamma\|y\|^{2} \leq C\left(M_{2}\right) .
$$

Notice that

$$
\|\nabla y\|^{2} \geq c_{0} N^{2}\|y\|^{2}
$$


then we can write

$$
\frac{\mathrm{d}}{\mathrm{d} t}\|y\|^{2}+2\left(c_{0} N^{2}-\gamma\right)\|y\|^{2} \leq C\left(M_{2}\right) .
$$

Let $N_{0}$ be large enough such that $c_{0} N_{0}^{2}-\gamma>0$. Then for $N \geq N_{0}$, multiplying the above inequality by $\mathrm{e}^{2\left(c_{0} N^{2}-\gamma\right) t}$, integrating it for $t$ from $t_{2}$ and using $y\left(t_{2}\right)=0$, we deduce

$$
\|y\|^{2} \leq \frac{C\left(M_{2}\right)}{2\left(c_{0} N^{2}-\gamma\right)} \triangleq K_{0}^{2}, \quad \forall t \geq t_{2}
$$

Note that the real part of the inner product of (3.1) with $-\triangle y$ is

$$
\frac{1}{2} \frac{\mathrm{d}}{\mathrm{d} t}\|\nabla y\|^{2}+\|\Delta y\|^{2}=\gamma\|\nabla y\|^{2}+\operatorname{Re}\left((1+\mathrm{i} \mu)\left(Q_{N}\left(|u|^{2 \sigma}(p+y)\right), \triangle y\right)\right) .
$$

By using the Sobolev interpolation inequality and inequality (3.8), we have the following estimates for the last two terms in the above expression:

$$
\begin{aligned}
& \mid \operatorname{Re}\left((1+\mathrm{i} \mu)\left(Q_{N}\left(|u|^{2 \sigma}(p+y), \triangle y\right)\right) \mid \leq \frac{1}{4}\|\triangle y\|^{2}+C\left(K_{0}, M_{2}\right),\right. \\
& \gamma\|\nabla y\|^{2} \leq \gamma\|y\|\|\triangle y\| \leq \frac{1}{4}\|\triangle y\|^{2}+\gamma^{2}\|y\|^{2} \leq \frac{1}{4}\|\triangle y\|^{2}+\gamma^{2} K_{0}^{2} .
\end{aligned}
$$

Thus, equation (3.9) can be rewritten as

$$
\frac{\mathrm{d}}{\mathrm{d} t}\|\nabla y\|^{2}+\|\triangle y\|^{2} \leq C\left(K_{0}, M_{2}\right)
$$

Noticing that $\|\triangle y\|^{2} \geq c N^{2}\|\nabla y\|^{2}$, we derive

$$
\frac{\mathrm{d}}{\mathrm{d} t}\|\nabla y\|^{2}+c N^{2}\|\nabla y\|^{2} \leq C\left(K_{0}, M_{2}\right) \text {. }
$$

Multiplying the above inequality by $\mathrm{e}^{c N^{2} t}$, integrating it for $t$ from $t_{2}$ and using $y\left(t_{2}\right)=0$, we have

$$
\|\nabla y\|^{2} \leq \frac{C\left(M_{0}, K_{0}\right)}{c N^{2}} \triangleq K_{1}^{2}, \quad \forall t \geq t_{2} .
$$

Since the real part of the inner product of (3.1) with $\triangle^{2} y$ is

$$
\frac{1}{2} \frac{\mathrm{d}}{\mathrm{d} t}\|\triangle y\|^{2}+\|\nabla \triangle y\|^{2}=\gamma\|\triangle y\|^{2}-\operatorname{Re}\left((1+\mathrm{i} \mu)\left(Q_{N}\left(|u|^{2 \sigma}(p+y)\right), \triangle^{2} y\right)\right),
$$

we can estimate the last two terms in (3.11) for $\sigma \geq \frac{1}{2}$. By using Hölder's inequality, the Sobolev interpolation inequality together with (3.8) and (3.10), we can derive the following estimates:

$$
\begin{gathered}
\left|\operatorname{Re}\left((1+\mathrm{i} \mu)\left(Q_{N}\left(|u|^{2 \sigma}(p+y)\right), \triangle^{2} y\right)\right)\right| \leq \frac{1}{4}\|\nabla \triangle y\|^{2}+C\left(K_{0}, K_{1}, M_{2}\right), \\
\gamma\|\triangle y\|^{2} \leq \gamma\|\nabla y\|\|\nabla \triangle y\| \leq \frac{1}{4}\|\nabla \triangle y\|^{2}+\gamma^{2}\|\nabla y\|^{2} \leq \frac{1}{4}\|\nabla \triangle y\|^{2}+\gamma^{2} K_{1}^{2} .
\end{gathered}
$$

Thus, equation (3.11) can be rewritten as

$$
\frac{\mathrm{d}}{\mathrm{d} t}\|\triangle y\|^{2}+\|\nabla \triangle y\|^{2} \leq C\left(K_{0}, K_{1}, M_{2}\right) .
$$

Thanks to $\|\nabla \triangle y\|^{2} \geq c N^{2}\|\triangle y\|^{2}$, we have

$$
\frac{\mathrm{d}}{\mathrm{d} t}\|\triangle y\|^{2}+c N^{2}\|\triangle y\|^{2} \leq C\left(K_{0}, K_{1}, M_{2}\right) .
$$


Multiplying (3.12) by $\mathrm{e}^{c N^{2} t}$, integrating it for $t$ from $t_{2}$ and using $y\left(t_{2}\right)=0$, we have

$$
\|\triangle y\|^{2} \leq \frac{C\left(K_{0}, K_{1}, M_{2}\right)}{c N^{2}} \triangleq \widetilde{K}^{2}, \quad \forall t \geq t_{2}
$$

If we choose $K_{2}=\sqrt{K_{0}^{2}+K_{1}^{2}+\widetilde{K}_{2}^{2}}$, then (3.6) holds for $m=2$.

Step 2: estimate for $y$ in $H_{p}^{m}(\Omega)(m \geq 3)$.

In this step, we prove that (3.6) holds for any $m \geq 3$ by using mathematical induction.

For $m=3$, differentiating (3.1) with respect to $t$ and taking the real part of the $L^{2}$-inner product with $-\triangle y_{t}$, we have

(3.13) $\frac{1}{2} \frac{\mathrm{d}}{\mathrm{d} t}\left\|\nabla y_{t}\right\|^{2}+\left\|\Delta y_{t}\right\|^{2}=\gamma\left\|\nabla y_{t}\right\|^{2}-\operatorname{Re}\left((1+\mathrm{i} \mu)\left(Q_{N}\left(|u|^{2 \sigma}(p+y)\right)_{t},-\triangle y_{t}\right)\right)$.

For $t \geq t_{2}$, by using Hölder's inequality, the Sobolev interpolation inequality and Lemma 2.2, we estimate the last two terms in (3.13) as follows:

$$
\begin{gathered}
\left|\operatorname{Re}\left((1+\mathrm{i} \mu)\left(Q_{N}\left(|u|^{2 \sigma}(p+y)\right)_{t},-\triangle y_{t}\right)\right)\right| \leq \frac{1}{4}\left\|\triangle y_{t}\right\|^{2}+C\left(K_{2}, M_{2}\right), \\
\gamma\left\|\nabla y_{t}\right\|^{2} \leq \frac{1}{4}\left\|\triangle y_{t}\right\|^{2}+\gamma^{2}\left\|y_{t}\right\|^{2} \leq \frac{1}{4}\left\|\triangle y_{t}\right\|^{2}+C\left(K_{2}, M_{2}\right) .
\end{gathered}
$$

Thus, equation (3.13) can be rewritten as

$$
\frac{\mathrm{d}}{\mathrm{d} t}\left\|\nabla y_{t}\right\|^{2}+\left\|\triangle y_{t}\right\|^{2} \leq C\left(K_{2}, M_{2}\right)
$$

Due to the inequality $\left\|\triangle y_{t}\right\|^{2} \geq c N^{2}\left\|\nabla y_{t}\right\|^{2}$ (see Lemma 3.1), the above expression can be rewritten as

$$
\frac{\mathrm{d}}{\mathrm{d} t}\left\|\nabla y_{t}\right\|^{2}+c N^{2}\left\|\nabla y_{t}\right\|^{2} \leq C\left(K_{2}, M_{2}\right) .
$$

Multiplying (3.14) by $\mathrm{e}^{c N^{2} t}$, integrating it for $t$ from $t_{2}$ and using $\left\|\nabla y\left(t_{2}\right)\right\| \leq$ $c\left\|u\left(t_{2}\right)\right\|_{2}^{2 \sigma+1} \leq C\left(M_{2}\right)$, we have

$$
\begin{aligned}
\left\|\nabla y_{t}\right\|^{2} & \leq \mathrm{e}^{-c N^{2}\left(t-t_{2}\right)}\left\|\nabla y_{t}\left(t_{2}\right)\right\|^{2}+\frac{C\left(K_{2}, M_{2}\right)}{c N^{2}}, \\
& \leq \mathrm{e}^{-c N^{2}\left(t-t_{2}\right)} C\left(M_{2}\right)+\frac{C\left(K_{2}, M_{2}\right)}{c N^{2}} \triangleq \rho_{3}^{2}, \quad \forall t \geq t_{2},
\end{aligned}
$$

which implies

$$
\|\nabla \triangle y\| \leq\left\|\nabla y_{t}\right\|+C_{0}\left(M_{2}, K_{2}\right) \leq \rho_{3}+C\left(M_{2}, K_{2}\right) \triangleq \widetilde{K}_{3}, \quad \forall t \geq t_{3}=t_{2}+1 .
$$

If we choose $K_{3}=\sqrt{K_{2}^{2}+\widetilde{K}_{3}^{2}}$, then (3.6) holds for $m=3$.

Suppose that (3.6) holds for any $3<m \leq k-1$, where $k$ is a positive integer. Namely, there exists constants $K_{m}$ such that

$$
\|y\|_{m} \leq K_{m}, \quad \forall t \geq t_{m}, \quad m \leq k-1 .
$$

Then it is yielded by using the Sobolev interpolation inequality that

$$
\|y\|_{W^{m-2, \infty}} \leq K_{m}^{\prime}, \quad \forall t \geq t_{m}, \quad m \leq k-1,
$$

where the constant $K_{m}^{\prime}$ depends on $K_{m}$. In addition, from Lemma 2.2 and the definition of $P_{N}$, we also have

$$
\|p\|_{m} \leq M_{m}, \quad\|p\|_{W^{m-2, \infty}} \leq C\left(K_{m}^{\prime}\right), \quad \forall t \geq t_{m}, \quad m \leq k-1,
$$


where $t_{m}$ in (3.15)-(3.17) is the same as given in Lemma 2.2.

Next we prove that (3.6) holds for $m=k$.

Taking $l=\left[\frac{k-1}{2}\right]$ and differentiating (3.1) for $l$ times with respect to $t$, we have

$$
y_{t^{l+1}}-(1+\mathrm{i} \nu) \triangle y_{t^{l}}+(1+\mathrm{i} \mu)\left(|u|^{2 \sigma}(p+y)\right)_{t^{l}}-\gamma y_{t^{l}}=0,
$$

If $k=2 l+1$ is an odd number, then taking the real part of the inner product of (3.18) with $-\triangle y_{t^{l}}$, we deduce

$$
\frac{1}{2} \frac{\mathrm{d}}{\mathrm{d} t}\left\|\nabla y_{t^{l}}\right\|^{2}+\left\|\triangle y_{t^{l}}\right\|^{2}=\gamma\left\|\nabla y_{t^{l}}\right\|^{2}+\operatorname{Re}\left((1+\mathrm{i} \mu) \int_{\Omega}\left(|u|^{2 \sigma}(p+y)\right)_{t^{l}} \triangle \bar{y}_{t^{l}} \mathrm{~d} x\right) .
$$

We can estimate the last two terms in (3.19) by using Hölder's inequality, the Sobolev interpolation inequality and Young's inequality together with (3.15)-(3.17). For $t \geq t_{k-1}=t_{2 l}$ and $\sigma \geq \frac{l}{2}$ or a positive integer, we have

$$
\begin{gathered}
\left|\operatorname{Re}\left((1+\mathrm{i} \mu) \int_{\Omega}\left(|u|^{2 \sigma}(p+y)\right)_{t^{l}} \triangle \bar{y}_{t^{l}} \mathrm{~d} x\right)\right| \\
\leq \frac{1}{4}\left\|\triangle y_{t^{l}}\right\|^{2}+C\left(K_{k-1}, K_{k-1}^{\prime}, M_{k-1}, M_{k-1}^{\prime}\right), \\
\gamma\left\|\nabla y_{t^{l}}\right\|^{2} \leq \gamma\left\|\triangle y_{t^{l}}\right\|\left\|y_{t^{l}}\right\| \leq \frac{1}{4}\left\|\left.\triangle y_{t^{l}}\right|^{2}+\gamma^{2}\right\| y_{t^{l}} \|^{2} .
\end{gathered}
$$

Hence, equation (3.19) can be rewritten as

$$
\frac{\mathrm{d}}{\mathrm{d} t}\left\|\nabla y_{t^{l}}\right\|^{2}+\left\|\triangle y_{t^{l}}\right\|^{2} \leq C_{1}\left(K_{k-1}, K_{k-1}^{\prime}, M_{k-1}^{\prime}, M_{k-1}\right), \quad \forall t \geq t_{k-1} .
$$

Using an analogous argument as that of (3.20) and taking the inner product of (3.18) with $y_{t^{l}}$, we have

$$
\frac{\mathrm{d}}{\mathrm{d} t}\left\|y_{t^{l}}\right\|^{2}+\left\|\nabla y_{t^{l}}\right\|^{2} \leq C_{2}\left(K_{k-1}, K_{k-1}^{\prime}, M_{k-1}, M_{k-1}^{\prime}\right), \quad \forall t \geq t_{k-1} .
$$

When $t \geq t_{k-1}$, it is implied by (3.21) that

$$
\begin{aligned}
\int_{t}^{t+1}\left\|\nabla y_{t^{l}}(s)\right\|^{2} \mathrm{~d} s & \leq\left\|y_{t^{l}}(t)\right\|^{2}+C_{2}\left(K_{k-1}, K_{k-1}^{\prime}, M_{k-1}^{\prime}, M_{k-1}\right), \\
& \leq C\left(K_{k-1}\right)+C_{2}\left(K_{k-1}, K_{k-1}^{\prime}, M_{k-1}^{\prime}, M_{k-1}\right) \triangleq \alpha_{3} .
\end{aligned}
$$

On the other hand, we set

$$
\alpha_{2}=C_{1}\left(K_{k-1}, K_{k-1}^{\prime}, M_{k-1}^{\prime}, M_{k-1}\right) .
$$

Then, applying the uniform Gronwall's inequality to (3.20) yields

$$
\left\|\nabla y_{t^{l}}(t+1)\right\|^{2} \leq \alpha_{3}+\alpha_{2}, \quad \forall t \geq t_{k-1}
$$

which leads to

$$
\left\|\nabla^{k} y\right\|^{2}=\left\|\nabla^{2 l+1} y\right\|^{2} \leq C\left(\alpha_{2}, \alpha_{3}\right), \quad \forall t \geq t_{k-1}+1 .
$$

If we choose $M_{k}=\sqrt{C\left(\alpha_{2}, \alpha_{3}\right)+K_{k-1}^{2}}$ and $t_{k}=t_{k-1}+1$, then (3.5) holds for $m=k$.

If $k=2 l+2$ is an even number, taking the real parts of the inner products of (3.18) with $\triangle^{2} y_{t^{l}}$ and $-\triangle y_{t^{l}}$, respectively, and using an analogous argument as that for (3.20) and (3.21), we have

$$
\frac{\mathrm{d}}{\mathrm{d} t}\left\|\triangle y_{t^{l}}\right\|^{2}+\left\|\nabla \triangle y_{t^{l}}\right\|^{2} \leq C_{1}\left(K_{k-1}, K_{k-1}^{\prime}, M_{k-1}^{\prime}, M_{k-1}\right), \quad \forall t \geq t_{k-1},
$$




$$
\frac{\mathrm{d}}{\mathrm{d} t}\left\|\nabla y_{t^{l}}\right\|^{2}+\left\|\triangle y_{t^{l}}\right\|^{2} \leq C_{2}\left(K_{k-1}, K_{k-1}^{\prime}, M_{k-1}, M_{k-1}^{\prime}\right), \quad \forall t \geq t_{k-1},
$$

respectively. Using the same procedure as the deduction of (3.22), we have

$$
\left\|\nabla^{k} y\right\|^{2}=\left\|\nabla^{2 l+2} y\right\|^{2} \leq C^{\prime}\left(\alpha_{2}^{\prime}, \alpha_{3}^{\prime}\right), \quad \forall t \geq t_{k-1}+1 .
$$

If we choose $K_{k}=\sqrt{C^{\prime}\left(\alpha_{2}^{\prime}, \alpha_{3}^{\prime}\right)+K_{k-1}^{2}}$ and $t_{k}=t_{k-1}+1$, then (3.5) holds for $m=k$.

Step 3: estimate for $z$ in $H_{p}^{2}(\Omega)$.

Taking the real part of the inner product of (3.3) with $z$ and recalling the definition of $Q_{N}$, we have

$$
\frac{\mathrm{d}}{\mathrm{d} t}\|z\|^{2}+2\|\nabla z\|^{2}+2 \int_{\Omega}|u|^{2 \sigma}|z|^{2} \mathrm{~d} x-2 \gamma\|z\|^{2}=0 .
$$

Since Lemma 3.1 implies

$$
\|\nabla z\|^{2} \geq c_{0} N^{2}\|z\|^{2}
$$

equation (3.23) can be reexpressed as

$$
\frac{\mathrm{d}}{\mathrm{d} t}\|z\|^{2}+2\left(c_{0} N^{2}-\gamma\right)\|z\|^{2} \leq 0
$$

Let $N_{1} \geq N_{0}$ be large enough such that $c_{0} N_{1}^{2}-\gamma>2 \mathrm{e}^{-1}$. Thus for $N \geq N_{1}$, multiplying the above inequality with $\mathrm{e}^{\lambda_{1} t}$ and integrating it for $t$ from $t_{2}$ gives

$$
\|z(t)\|^{2} \leq\left\|z\left(t_{2}\right)\right\|^{2} \mathrm{e}^{-\lambda_{1}\left(t-t_{2}\right)}, \quad \forall t \geq t_{2},
$$

where $\lambda_{1}=\lambda_{1}(N)=2\left(c_{0} N^{2}-\gamma\right)>2 \mathrm{e}^{-1}$.

Taking the real part of the inner product of (3.3) with $-\triangle z$ yields

$$
\frac{1}{2} \frac{\mathrm{d}}{\mathrm{d} t}\|\nabla z\|^{2}+\|\triangle z\|^{2}-\gamma\|\nabla z\|^{2}=\operatorname{Re}\left((1+\mathrm{i} \mu) \int_{\Omega} Q_{N}\left(|u|^{2 \sigma} z\right) \triangle \bar{z} \mathrm{~d} x\right) .
$$

For the last two terms in the above expression, by using Hölder's inequality and Young's inequality we obtain

$$
\begin{aligned}
\gamma\|\nabla z\|^{2} \leq \frac{1}{4} \| \triangle & \left\|^{2}+\gamma^{2}\right\| z \|^{2}, \\
\operatorname{Re}\left((1+\mathrm{i} \mu) \int_{\Omega} Q_{N}\left(|u|^{2 \sigma} z\right) \triangle \bar{z} \mathrm{~d} x\right) & \leq \frac{1}{4}\|\triangle z\|^{2}+\left(1+\mu^{2}\right)\|u\|_{\infty}^{4 \sigma}\|z\|^{2}, \\
& \leq \frac{1}{4}\|\triangle z\|^{2}+c M_{2}^{4 \sigma}\|z\|^{2} .
\end{aligned}
$$

Thus, (3.25) can be reexpressed as

$$
\frac{\mathrm{d}}{\mathrm{d} t}\|\nabla z\|^{2}+\|\triangle z\|^{2} \leq 2\left(\gamma^{2}+c M_{2}^{4 \sigma}\right)\|z\|^{2} .
$$

Inserting the inequality $\|\triangle z\|^{2} \geq c_{1} N^{2}\|\nabla z\|^{2}$ (see Lemma 3.2) into the above expression yields

$$
\frac{\mathrm{d}}{\mathrm{d} t}\|\nabla z\|^{2}+c_{1} N^{2}\|\nabla z\|^{2} \leq 2\left(\gamma^{2}+c M_{2}^{4 \sigma}\right)\|z\|^{2} .
$$

Let $N_{2} \geq N_{1}$ be large enough such that $c_{1} N_{2}^{2}>2 \mathrm{e}^{-1}$. Thus for $N \geq N_{2}$, multiplying the above inequality by $\mathrm{e}^{c_{1} N^{2} t}$, integrating it for $t$ from $t_{2}$ and using (3.24), we 
derive

$$
\begin{aligned}
\|\nabla z\|^{2} & \leq\left\|\nabla z\left(t_{2}\right)\right\|^{2} \mathrm{e}^{-c_{1} N^{2}\left(t-t_{2}\right)}+2\left(\gamma^{2}+c M_{2}^{4 \sigma}\right) M_{2}^{2}\left(t-t_{2}\right) \mathrm{e}^{-\lambda_{2}^{\prime}\left(t-t_{2}\right)}, \\
& \leq M_{2}^{2} \mathrm{e}^{-c_{1} N^{2}\left(t-t_{2}\right)}+2\left(\gamma^{2}+c M_{2}^{4 \sigma}\right) M_{2}^{2} \mathrm{e}^{-\left(\lambda_{2}^{\prime}-\mathrm{e}^{-1}\right)\left(t-t_{2}\right)}, \\
& \leq\left(2\left(\gamma^{2}+c M_{2}^{4 \sigma}\right)+1\right) M_{2}^{2} \mathrm{e}^{-\left(\lambda_{2}^{\prime}-\mathrm{e}^{-1}\right)\left(t-t_{2}\right)}, \\
& \triangleq C_{1}\left(M_{2}\right) \mathrm{e}^{-\lambda_{2}\left(t-t_{2}\right)}, \quad \forall t \geq t_{2},
\end{aligned}
$$

where $\lambda_{2}^{\prime}=\min \left\{\lambda_{1}, c_{1} N^{2}\right\}, \mathrm{e}^{-1}<\lambda_{2}=\lambda_{2}^{\prime}-\mathrm{e}^{-1}<\lambda_{1}$ and $C_{1}\left(M_{2}\right)=\left(2\left(\gamma^{2}+\right.\right.$ $\left.\left.c M_{2}^{4 \sigma}\right)+1\right) M_{2}^{2}$.

Taking the real part of the inner product of (3.1) with $\triangle^{2} z$ gives

$$
\frac{1}{2} \frac{\mathrm{d}}{\mathrm{d} t}\|\triangle z\|^{2}+\|\nabla \triangle z\|^{2}-\gamma\|\triangle z\|^{2}=-\operatorname{Re}\left((1+\mathrm{i} \mu) \int_{\Omega} Q_{N}\left(|u|^{2 \sigma} z\right) \triangle^{2} \bar{z} \mathrm{~d} x\right) .
$$

For the last two terms in equation (3.27), again using Hölder's inequality and Young's inequality we can deduce

$$
\begin{gathered}
\operatorname{Re}\left((1+\mathrm{i} \mu) \int_{\Omega} Q_{N}\left(|u|^{2 \sigma} z\right) \triangle^{2} \bar{z} \mathrm{~d} x\right) \leq \frac{1}{4}\|\nabla \triangle z\|^{2}+c M_{2}^{4 \sigma}\left(\|\nabla z\|^{2}+\|z\|^{2}\right) \\
\gamma\|\triangle z\|^{2} \leq \frac{1}{4}\|\nabla \triangle z\|^{2}+\gamma^{2}\|\nabla z\|^{2}
\end{gathered}
$$

From Lemma 3.1 we have

$$
\|\nabla \triangle z\|^{2} \geq c_{2} N^{2}\|\triangle z\|^{2}
$$

Then (3.27) can be reexpressed as

$$
\frac{\mathrm{d}}{\mathrm{d} t}\|\triangle z\|^{2}+c_{2} N^{2}\|\triangle z\|^{2} \leq 2 \gamma^{2}\|\nabla z\|^{2}+c M_{2}^{4 \sigma}\|z\|_{1}^{2} .
$$

Let $N_{3} \geq N_{2}$ be large enough such that $c_{2} N_{3}^{2}>\mathrm{e}^{-1}$. Thus for $N \geq N_{3}$, multiplying the above inequality with $\mathrm{e}^{c_{2} N^{2} t}$, integrating it for $t$ from $t_{2}$ and using (3.24)-(3.26), we deduce

$$
\begin{aligned}
\|\Delta z\|^{2} \leq & \left\|\Delta z\left(t_{2}\right)\right\|^{2} \mathrm{e}^{-c_{2} N^{2}\left(t-t_{2}\right)} \\
& +c M_{2}^{4 \sigma+2} \frac{\mathrm{e}^{\lambda_{1} t_{2}-c_{2} N^{2} t}}{c_{2} N^{2}-\lambda_{1}}\left(\mathrm{e}^{\left(c_{2} N^{2}-\lambda_{1}\right) t}-\mathrm{e}^{\left(c_{2} N^{2}-\lambda_{1}\right) t}\right) \\
& +\left(2 \gamma^{2}+c M_{2}^{4 \sigma}\right) C_{1}\left(M_{2}\right) \frac{\mathrm{e}^{\lambda_{2} t_{2}-c_{2} N^{2} t}}{c_{2} N^{2}-\lambda_{2}}\left(\mathrm{e}^{\left(c_{2} N^{2}-\lambda_{2}\right) t}-\mathrm{e}^{\left(c_{2} N^{2}-\lambda_{2}\right) t}\right), \\
\leq & M_{2}^{2} \mathrm{e}^{-c_{2} N^{2}\left(t-t_{2}\right)}+\left(\left(2 \gamma^{2}+c M_{2}^{4 \sigma}\right) C_{1}\left(M_{2}\right)\right. \\
& \left.+c M_{2}^{4 \sigma+2}\right)\left(t-t_{2}\right) \mathrm{e}^{-\lambda_{3}^{\prime}\left(t-t_{2}\right)}, \\
\leq & \left(M_{2}^{2}+\left(2 \gamma^{2}+c M_{2}^{4 \sigma}\right) C_{1}\left(M_{2}\right)+c M_{2}^{4 \sigma+2}\right) \mathrm{e}^{-\left(\lambda_{3}^{\prime}-\mathrm{e}^{-1}\right)\left(t-t_{2}\right)}, \\
\triangleq & C_{2}\left(M_{2}\right) \mathrm{e}^{-\lambda_{3}\left(t-t_{2}\right)}, \quad \forall t \geq t_{2},
\end{aligned}
$$

where $\lambda_{3}^{\prime}=\min \left\{c_{2} N^{2}, \lambda_{1}, \lambda_{2}\right\}, \lambda_{3}=\lambda_{3}^{\prime}-\mathrm{e}^{-1}$ and $C_{2}\left(M_{2}\right)=M_{2}^{2}+\left(2 \gamma^{2}+\right.$ $\left.c M_{2}^{4 \sigma}\right) C_{1}\left(M_{2}\right)+c M_{2}^{4 \sigma+2}$.

Let $\lambda=\frac{1}{2} \min \left(\lambda_{1}, \lambda_{2}, \lambda_{3}\right)$ and $C\left(M_{2}\right)=\max \left\{M_{2}, C_{1}\left(M_{2}\right), C_{2}\left(M_{2}\right)\right\}$. Then combining $(3.24),(3.26)$ and (3.28), we can see that the estimate (3.6) holds. Therefore, we have completed the proof of Theorem 3.2. 
In virtue of Theorem 3.2, the solution operator $S(t)=S^{(2)}(t): H_{p}^{2}(\Omega) \rightarrow$ $H_{p}^{2}(\Omega)$ generated by problem (1.2)-(1.4) can be decomposed as

$$
S^{(2)}(t)=S_{1}^{(2)}(t)+S_{2}^{(2)}(t), \quad \forall t \geq 0,
$$

where $S_{1}^{(2)}(t)$ and $S_{2}^{(2)}(t)$ are defined by

and

$$
S_{1}^{(2)}(t) u_{0}= \begin{cases}P_{N} u(t)+y(t)=p(t)+y(t), & t \geq t_{2}, \\ P_{N} u(t)=p(t), & t \leq t_{2},\end{cases}
$$

$$
S_{2}^{(2)}(t) u_{0}= \begin{cases}z(t), & t \geq t_{2}, \\ Q_{N} u(t)=q(t), & t \leq t_{2},\end{cases}
$$

where $u(t)=S^{(2)} u_{0}$, and for $t \geq t_{2}, y(t)$ and $z(t)$ are solutions of (3.1) and (3.2), and (3.3) and (3.4), respectively.

Hence, for every $u \in H_{p}^{2}(\Omega)$, we have

$$
S^{(2)}(t) u=S_{1}^{(2)}(t) u+S_{2}^{(2)}(t) u
$$

\section{Regularity of Attractor}

In this section, we will present our main result on regularity of attractor.

THEOREM 4.1. Suppose that condition (A3) holds and $\sigma$ is a positive integer or $\sigma \geq \frac{1}{2}\left(\left[\frac{m}{2}\right]\right)$ for any positive integer $m \geq 2$. Let $\mathcal{A}_{m}$ be the global attractors of semi-group of the operators, $\left\{S^{(m)}(t)\right\}_{t \geq 0}$, generated by (1.2). Then we have the following results:

(i) For any $m \geq 3, \mathcal{A}_{2}$ is a bounded closed set in $H_{p}^{m}(\Omega)$.

(ii) $\mathcal{A}_{2}=\mathcal{A}_{m}$ for $m \geq 3$.

ProOf. (i) Suppose that $u \in \mathcal{A}_{2}$. We shall prove $u \in H_{p}^{m}(\Omega)$ for any $m \geq 3$.

Due to a well-known characterization of $\omega$-limit sets (see [18]), there exist a sequence of elements $u_{n}$ in $B_{2}$ and a sequence of positive real numbers $t_{n}^{\prime}$ which approaches infinity as $n$ tends to infinity such that

$$
S^{(2)}\left(t_{n}^{\prime}\right) u_{n} \rightarrow u \text { in } H_{p}^{2}(\Omega), \text { as } n \rightarrow+\infty .
$$

We also have from (3.29) that

$$
S^{(2)}\left(t_{n}^{\prime}\right) u_{n}=S_{1}^{(2)}\left(t_{n}^{\prime}\right) u_{n}+S_{2}^{(2)}\left(t_{n}^{\prime}\right) u_{n}, \quad \forall n \in \mathbf{N} .
$$

From the definitions of $S_{1}^{(2)}(t)$ and $S_{2}^{(2)}(t)$, using Theorem 3.1 and $\|p(t)\|_{2} \leq M_{2}$, we deduce that if $N$ is large enough (as given in Theorem 3.1), then

$$
\left\|S_{1}^{(2)}\left(t_{n}^{\prime}\right) u_{n}\right\|_{m} \leq C(N, m), \quad \forall n \in \mathbf{N},
$$

and

$$
\left\|S_{2}^{(2)}\left(t_{n}^{\prime}\right) u_{n}\right\|_{2} \leq C\left(M_{2}\right) \mathrm{e}^{-\lambda t_{n}^{\prime}}, \quad \forall n \in \mathbf{N} .
$$

Thanks to (4.3) we infer that there exist subsequences $\left\{t_{n^{\prime}}^{\prime}\right\}_{n^{\prime}>0}$ and $w \in$ $H_{p}^{m}(\Omega)$ such that

$$
S_{1}^{(2)}\left(t_{n^{\prime}}^{\prime}\right) u_{n^{\prime}} \rightarrow w \quad \text { weakly in } H_{p}^{m}(\Omega), \quad \text { as } n^{\prime} \rightarrow \infty,
$$

and

$$
\|w\|_{m} \leq \lim _{n^{\prime} \rightarrow \infty} \inf \left\|S_{1}^{(2)}\left(t_{n^{\prime}}^{\prime}\right) u_{n^{\prime}}\right\|_{m} \leq C(N, m)
$$


By taking account of $\varphi \in L_{p}^{2}(\Omega)$, it is implied from (4.2) that

$$
\left(S^{(2)}\left(t_{n^{\prime}}^{\prime}\right)\left(u_{n^{\prime}}\right), \varphi\right)=\left(S_{1}^{(2)}\left(t_{n^{\prime}}^{\prime}\right)\left(u_{n^{\prime}}\right), \varphi\right)+\left(S_{2}^{(2)}\left(t_{n^{\prime}}^{\prime}\right)\left(u_{n^{\prime}}\right), \varphi\right) .
$$

Taking the limit $n^{\prime} \rightarrow+\infty$ in the above expression and using (4.1), (4.4) and (4.5), we deduce

$$
(u, \varphi)=(w, \varphi), \quad \forall \varphi \in L_{p}^{2}(\Omega) .
$$

Setting $\varphi=(-\triangle)^{m} u$ in the above expression and using (4.6), we derive

$$
\left\|\nabla^{m} u\right\| \leq\|w\|_{m} \leq C(N, m),
$$

which shows $u \in H_{p}^{m}(\Omega)$. In other words, $\mathcal{A}_{2}$ is a bounded set in $H_{p}^{m}(\Omega)$.

(ii) On the one hand, we prove that $\mathcal{A}_{2} \subset \mathcal{A}_{m}$.

Since $\mathcal{A}_{m}$ attracts all bounded sets in $H_{p}^{m}(\Omega)$ and $\mathcal{A}_{2}$ is bounded in $H_{p}^{m}(\Omega)$ (due to (i)), we have

$$
\operatorname{dist}_{H_{p}^{m}(\Omega)}\left(S^{(2)}(t) \mathcal{A}_{2}, \mathcal{A}_{m}\right)=\operatorname{dist}_{H_{p}^{m}(\Omega)}\left(S^{(m)}(t) \mathcal{A}_{2}, \mathcal{A}_{m}\right) \rightarrow 0, \quad \text { as } t \rightarrow \infty .
$$

In addition, $\mathcal{A}_{2}$ is an invariant set of $S^{(2)}(t)$, namely, $S^{(2)}(t) \mathcal{A}_{2}=\mathcal{A}_{2}$, which leads to

$$
\operatorname{dist}_{H_{p}^{m}(\Omega)}\left(\mathcal{A}_{2}, \mathcal{A}_{m}\right)=0 .
$$

Hence, by virtue of the fact that $\mathcal{A}_{m}$ is closed in $H_{p}^{m}(\Omega)$, we have

$$
\mathcal{A}_{2} \subset \mathcal{A}_{m} \text {. }
$$

On the other hand, we show that $\mathcal{A}_{m} \subset \mathcal{A}_{2}$. Since $\mathcal{A}_{2}$ attracts the bounded set $\mathcal{A}_{m}$ in $H_{p}^{2}(\Omega)$, we have

$$
\operatorname{dist}_{H_{p}^{2}(\Omega)}\left(S^{(m)}(t) \mathcal{A}_{m}, \mathcal{A}_{2}\right)=\operatorname{dist}_{H_{p}^{2}(\Omega)}\left(S^{(2)}(t) \mathcal{A}_{m}, \mathcal{A}_{2}\right) \rightarrow 0, \quad \text { as } t \rightarrow \infty .
$$

It is obtained by using $S^{(m)}(t) \mathcal{A}_{m}=\mathcal{A}_{m}$ and the Sobolev embedding theorem that

$$
\operatorname{dist}_{H_{p}^{2}(\Omega)}\left(\mathcal{A}_{m}, \mathcal{A}_{2}\right)=0,
$$

that is,

$$
\mathcal{A}_{m} \subset \mathcal{A}_{2} \text {. }
$$

Therefore, the proof of Theorem 4.1 is completed.

\section{References}

[1] J.M Ghidaglia and B. Héron, Dimension of the attractor associated to the Ginzburg-Landau equation, Phys. D 28 (1987), 282-304.

[2] C.R. Doering, J.D. Gibbon, D. Holm and B. Nicolaenko, Low-dimensional behavior in the complex Ginzburg-Landau equation, Nonlinearity 1(1988), 279-309.

[3] K. Promislow, Induced trajectories and approximate inertial manifolds for the GinzburgLandau partial differential equation, Phys. D 41 (1990), 232-252.

[4] C. Bu, On the Cauch problem for the 1+2 complex Ginzburg-Landau equation, J. Austral Math. Soc. Ser. B 36 (1994), 313-324.

[5] S.J. Lü, The dynamical behavior of the Ginzburg-Landau equation and its Fourier spectral approximation, Numerical Math. 22 (2000), 1-9.

[6] B.L. Guo and B.X. Wang, Finite dimensional behavior for the derivative Ginzburg-Landau equation in two spatial dimensions, Phys. D 89 (1995), 83-99.

[7] Y.S. Li and B.L. Guo, Global existence of solutions to the derivative 2D Ginzburg-Landau equation, J. Math. Anal. Appl. 249 (2000), 412-432.

[8] H.J. Gao and J.Q. Duan, On the initial value problem for the generalized 2D GinzburgLandau equation, J. Math. Anal. Appl. 216 (1997), 536-548.

[9] H.J. Gao and J.Q. Duan, Asymptotics for the generalized two-dimensional Ginzburg-Landau equation, J. Math. Anal. Appl. 247 (2000), 198-216. 
[10] C.R. Doering, J.D. Gibbon and C.D. Levermore, Weak and strong solutions of the complex Ginzburg-Landau equation, Phys. D 71 (1994), 285-318.

[11] N. Okazawa and T. Yokota, Monotonicity method applied to the complex Ginzburg-Landau and related equations, J. Math. Anal. Appl. 267 (2002), 247-263.

[12] S.J. Lü and Q.S. Lu, Exponential attractor for the 3D Ginburg-Landau type equation, Nonl. Anal. 67 (2007), 3116-3135.

[13] S.J. Lü and Q.S. Lu, Fourier spectral approximation to long-time behavior of three dimensional Ginzburg-Landau type equation, Adv. Comp. Math. 27 (2007), 293-318.

[14] T. Tao, A global compact attractor for high-dimensional defocusing non-linear schrödinger equations with potential, Dyn. Partial Differ. Equ, 5 (2008), 101-116.

[15] T. Tao, On the asymptotic behavior of large radial data for a focusing non-linear schrödinger equation, Dyn. Partial Differ. Equ, 1 (2004), 1-48.

[16] V.G. Maeja, Sobolev Space, Springer-Verlag, New York, 1985.

[17] J. Shen, Long-time stability and convergence for fully discrete nonlinear Galerkin methods, Appl. Anal. 38 (1990), 201-209.

[18] R. Temam, Infinite Dimensional Dynamical Systems in Mechanics and Physics, SpringerVerlag, New York, 1995.

[19] C. Canuto and A. Qarteroni, Approximation results for orthogonal polynomials in Sobolev spaces, Math. Comp. 38 (1982), 67-86.

Shujuan Lü, Qishao Lu: Department of Mathematics \& LMiB, Beijing University of Aeronautics And Astronautics, Beijing 100083, China

Qingguo Meng: Department of Mechanics, Tianjin University of Technology and Education, Tianjin 300222, China

Zhaosheng Feng: Corresponding author, Department of Mathematics, University of Texas-Pan American, Edinburg, TX 78539, USA

E-mail address: zsfeng@utpa.edu 